\title{
Stabilizability of Linear Impulsive Systems
}

\author{
Douglas A. Lawrence
}

\begin{abstract}
This paper establishes the equivalence of three stabilizability-related properties for a class of linear impulsive systems. The first involves a gramian-based condition inspired by results for time-varying, discrete-time linear systems introduced decades ago. The second is the ability to achieve closed-loop exponential stability via state feedback. Finally, the third property is exponential stability of an 'unreachable' subsystem identified from a decomposition of the original system derived from an invariant subspace that characterizes the set of reachable states. A consequence of this analysis is that full state reachability of a linear impulsive system is not necessary for state feedback stabilization, a well-known fact for linear time-invariant systems. The main ideas of the paper are applied to the problem of synchronizing two Lorenz oscillators using underactuated impulsive control.
\end{abstract}

\section{INTRODUCTION}

Stabilizability and its dual, detectability, are linear system properties of longstanding interest in connection with various feedback stabilization problems. In the linear timeinvariant (LTI) case, the well-known link between controllability/reachability and eigenvalue placement by state feedback together with the ability to decompose an LTI state equation into 'controllable' and 'uncontrollable' subsystems lead to the conclusion that stabilizability by state feedback is equivalent to exponential stability of the uncontrollable subsystem. A generalization of this result to time-varying linear systems, for which the aforementioned decomposition is usually unavailable, is presented in [1] for the discrete-time case. In this work, uniform stabilizability, loosely defined as uniform positive definiteness of the reachability gramian along unstable trajectories, is shown to be necessary and sufficient for state feedback stabilization. The recent analysis in [3] sharpens the feedback gain construction so as to reduce its dependence on the system data, i.e., the sequence of state equation coefficient matrices.

Stabilizability has been recently addressed for switched linear systems, in both continuous-time [11] and discretetime [12] cases. The characterization of the set of reachable states in terms of a fixed invariant subspace permits a decomposition of a switched linear system comparable to that for LTI systems. An open-loop characterization of stabilizability is shown to hold if and only if the 'unreachable' subsystem is asymptotically stable. The concepts of uniform and causal finite-path stabilizability (and detectability) arise in the study of feedback stabilization for discrete-time switched linear systems in [4].

Here we present a stabilizability analysis for a class of impulsive linear systems that builds upon recent investigations

D. A. Lawrence is with the School of Electrical Engineering and Computer Science, Ohio University, Athens, OH, USA dal @ohio . edu into reachability properties [7], [9] and feedback stabilization [8]. As with the development in [11], [12], a linear impulsive state system can be decomposed into, loosely speaking, reachable and unreachable subsystems defined in terms of an invariant subspace associated with the set of reachable states. Following the geometric approach to LTI systems [10], these subsystems are referred to as the restriction of the linear impulsive system to the invariant subspace and the subsystem induced by the linear impulsive system on the quotient space associated with the invariant, respectively. Within this framework, a gramian-based characterization of stabilizability in the style of [1] and stabilizability by state feedback are each shown to be equivalent to exponential stability of the 'unreachable' subsystem under an additional technical assumption. Consequently, state feedback stabilization for linear impulsive systems does not require full state reachability, thereby generalizing a longstanding result for the LTI case.

The remainder of the paper is organized as follows. The class of linear impulsive systems under consideration is presented in Section II. Concepts related to the reachability and feedback stabilization of linear impulsive systems are reviewed in Section III. Three system properties related to stabilizability are presented in Section IV and shown to be equivalent under certain mild assumptions. The main ideas of the paper are applied to the problem of synchronizing two Lorenz oscillators using impulsive control in Section V. Concluding remarks are presented in Section VI. Some of the details involving the construction of stabilizing state feedback laws are collected in an Appendix.

\section{LINEAR IMPULSIVE SySTEMS}

We consider linear impulsive systems described by state equations of the form

$$
\begin{aligned}
\dot{x}(t) & =A_{\mathcal{C}} x(t)+B_{\mathcal{C}} u(t) & t & \in \mathbb{R} \backslash \mathcal{T} \\
x\left(\tau_{k}\right) & =A_{\mathcal{I}} x\left(\tau_{k}^{-}\right)+B_{\mathcal{I}} w[k] & \tau_{k} & \in \mathcal{T}
\end{aligned}
$$

where $\mathcal{T}$ is a countably infinite set of strictly increasing impulse times, $x(t)$ is the continuous-time state that undergoes instantaneous changes at the impulse times, $u(t)$ is a continuous-time input assumed to be piecewise continuous, and $w[k]$ is a discrete-time input. For $\tau_{k} \in \mathcal{T}$, we denote $x\left(\tau_{k}^{-}\right)=\lim _{\epsilon \rightarrow 0^{+}} x\left(\tau_{k}-\epsilon\right)$ and $x\left(\tau_{k}^{+}\right)=\lim _{\epsilon \rightarrow 0^{+}} x\left(\tau_{k}+\epsilon\right)$. In our set-up, the impulsive state equation (1) produces rightcontinuous state trajectories, i.e., $x\left(\tau_{k}\right)=x\left(\tau_{k}^{+}\right), \tau_{k} \in \mathcal{T}$. The state space for (1) is denoted by $\mathcal{X}$ and $n:=\operatorname{dim}(\mathcal{X})$. With $\delta_{k}:=\tau_{k+1}-\tau_{k}$, we further assume: 
Assumption 2.1: The impulse time set $\mathcal{T}$ satisfies

$$
\underline{\delta}:=\inf _{k} \delta_{k}>0 \text { and } \bar{\delta}:=\sup _{k} \delta_{k}<\infty
$$

The lower bound ensures that $\tau_{k} \rightarrow \infty$ as $k \rightarrow \infty$ and that any finite time interval contains a finite number of impulse times. Both bounds are used in several technical arguments in the sequel.

\section{A. Notation and Preliminaries}

The notation $\|\cdot\|$ will be used to denote the Euclidean norm for vectors and the corresponding induced (spectral) norm for matrices. This allows us to write $\left\|A^{T}\right\|=\|A\|$ for any matrix $A$. To facilitate subsequent bookkeeping, we introduce two functions. First, $\kappa: \mathbb{R} \longrightarrow \mathcal{T}$ is defined by

$$
\kappa(t)=\sup \left\{k \in \mathbb{Z} \mid \tau_{k} \in \mathcal{T} \text { and } \tau_{k} \leq t\right\}
$$

For a given impulse time set $\mathcal{T}$ and integer $m$, we define the $\operatorname{map} \sigma_{m}: \mathbb{R} \longrightarrow \mathbb{R}$ via

$$
\sigma_{m}(t)=\tau_{\kappa(t)+m}+\frac{\delta_{\kappa(t)+m}}{\delta_{\kappa(t)}}\left(t-\tau_{\kappa(t)}\right)
$$

That is, $\sigma_{m}(t)$ defines a time instant separated from $t$ by $|m|$ impulse times that represents the same proportion of elapsed time with respect to the interval $\left[\tau_{\kappa(t)+m}, \tau_{\kappa(t)+m+1}\right)$ that $t$ does with respect to $\left[\tau_{\kappa(t)}, \tau_{\kappa(t)+1}\right)$. We observe that $\sigma_{0}(t)=t$ and $\sigma_{i}\left(\sigma_{j}(t)\right)=\sigma_{i}\left(\sigma_{j}(t)\right)=\sigma_{i+j}(t)$.

The state transition matrix for (1), denoted $\Phi(\cdot, \cdot)$, is characterized by $\Phi(t, t)=I$ and for $t>s$

$$
\begin{aligned}
\frac{\partial \Phi}{\partial t}(t, s) & =A_{\mathcal{C}} \Phi(t, s) & t & \in \mathbb{R} \backslash \mathcal{T} \\
\Phi\left(\tau_{k}, s\right) & =A_{\mathcal{I}} \Phi\left(\tau_{k}^{-}, s\right) & \tau_{k} & \in \mathcal{T}
\end{aligned}
$$

The linear impulsive system (1) is reversbible, i.e., $\Phi(t, s)$ is invertible for all $t$ and $s$, if and only if $A_{\mathcal{I}}$ is invertible in which case $\Phi^{-1}(t, s)=\Phi(s, t)$.

\section{REACHABILITY}

The set of reachable states for fixed initial time, final time, and impulse times is defined as

$$
\begin{array}{r}
\mathcal{R}_{\text {fixed }}\left(t_{0}, t_{f}, \mathcal{T}\right)=\left\{x_{f} \in \mathcal{X} \mid \exists \text { p.c. } u(\cdot) \text { and } w[\cdot]\right. \text { s.t. } \\
\left.x\left(t_{0}\right)=0 \text { and } x\left(t_{f}\right)=x_{f}\right\}
\end{array}
$$

$\mathcal{R}_{\text {fixed }}\left(t_{0}, t_{f}, \mathcal{T}\right)$ is also a subspace of $\mathcal{X}$ given by

$\mathcal{R}_{\text {fixed }}\left(t_{0}, t_{f}, \mathcal{T}\right)=\sum_{j=\kappa\left(t_{0}\right)+1}^{\kappa\left(t_{f}\right)} \Phi\left(t_{f}, \tau_{j}\right)\left(A_{\mathcal{I}} \mathcal{R}_{\mathcal{C}}+\mathcal{B}_{\mathcal{I}}\right)+\mathcal{R}_{\mathcal{C}}$

in which $\mathcal{R}_{\mathcal{C}}$ is the reachable subspace of the continuous-time pair $\left(A_{\mathcal{C}}, B_{\mathcal{C}}\right)$ that coincides with the smallest $A_{\mathcal{C}}$-invariant subspace containing $\mathcal{B}_{\mathcal{C}}:=\operatorname{Im} B_{\mathcal{C}}$, denoted $\left\langle A_{\mathcal{C}} \mid \mathcal{B}_{\mathcal{C}}\right\rangle$, and $\mathcal{B}_{\mathcal{I}}:=\operatorname{Im} B_{\mathcal{I}}$.
For the impulsive system (1), the reachability gramian $W\left(t_{0}, t_{f}\right)$ is defined as [8]

$$
\begin{aligned}
W\left(t_{0}, t_{f}\right)= & \int_{t_{0}}^{t_{f}} \Phi\left(t_{f}, \tau\right) B_{\mathcal{C}} B_{\mathcal{C}}^{T} \Phi^{T}\left(t_{f}, \tau\right) d \tau \\
& +\sum_{j=\kappa\left(t_{0}\right)+1}^{\kappa\left(t_{f}\right)} \Phi\left(t_{f}, \tau_{j}\right) B_{\mathcal{I}} B_{\mathcal{I}}^{T} \Phi^{T}\left(t_{f}, \tau_{j}\right)
\end{aligned}
$$

\section{A. Subsystems}

We let $\left\langle A_{\mathcal{C}}, A_{\mathcal{I}} \mid \mathcal{B}_{\mathcal{C}}+\mathcal{B}_{\mathcal{I}}\right\rangle$ denote the smallest subspace of $\mathcal{X}$ that is invariant with respect to both maps $A_{\mathcal{C}}$ and $A_{\mathcal{I}}$ and contains $\mathcal{B}_{\mathcal{C}}+\mathcal{B}_{\mathcal{I}}$. For the impulsive system (1), we define a pair of subsystems associated with $\left\langle A_{\mathcal{C}}, A_{\mathcal{I}} \mid \mathcal{B}_{\mathcal{C}}+\mathcal{B}_{\mathcal{I}}\right\rangle$. First, we regard $\left\langle A_{\mathcal{C}}, A_{\mathcal{I}} \mid \mathcal{B}_{\mathcal{C}}+\mathcal{B}_{\mathcal{I}}\right\rangle$ itself as a linear vector space of dimension less than or equal to $n=\operatorname{dim}(\mathcal{X})$, which, to avoid confusion, we denote by $\mathcal{R}$. We let $R: \mathcal{R} \rightarrow \mathcal{X}$ denote the insertion map that uniquely associates each element of the vector space $\mathcal{R}$ with an element of the vector space $\mathcal{X}$ that lies $\left\langle A_{\mathcal{C}}, A_{\mathcal{I}} \mid \mathcal{B}_{\mathcal{C}}+\mathcal{B}_{\mathcal{I}}\right\rangle$ [10]. In other words, $R \mathcal{R}=$ $\left\langle A_{\mathcal{C}}, A_{\mathcal{I}} \mid \mathcal{B}_{\mathcal{C}}+\mathcal{B}_{\mathcal{I}}\right\rangle$ or $\operatorname{Im} R=\left\langle A_{\mathcal{C}}, A_{\mathcal{I}} \mid \mathcal{B}_{\mathcal{C}}+\mathcal{B}_{\mathcal{I}}\right\rangle$.

As $\left\langle A_{\mathcal{C}}, A_{\mathcal{I}} \mid \mathcal{B}_{\mathcal{C}}+\mathcal{B}_{\mathcal{I}}\right\rangle$ is invariant with respect to both $A_{\mathcal{C}}$ and $A_{\mathcal{I}}$, each map has a well-defined restriction to $\mathcal{R}$, denoted $\hat{A}_{\mathcal{C}}$ and $\hat{A}_{\mathcal{I}}$, respectively. These maps satisfy the commutative relationships

$$
A_{\mathcal{C}} R=R \hat{A}_{\mathcal{C}} \quad \text { and } \quad A_{\mathcal{I}} R=R \hat{A}_{\mathcal{I}}
$$

In addition, since both $\mathcal{B}_{\mathcal{C}}$ and $\mathcal{B}_{\mathcal{I}}$ are contained in $\left\langle A_{\mathcal{C}}, A_{\mathcal{I}}\right.$ $\left.\mathcal{B}_{\mathcal{C}}+\mathcal{B}_{\mathcal{I}}\right\rangle$, there are well-defined maps $\hat{B}_{\mathcal{C}}$ and $\hat{B}_{\mathcal{I}}$ that satisfy

$$
B_{\mathcal{C}}=R \hat{B}_{\mathcal{C}} \quad \text { and } \quad B_{\mathcal{I}}=R \hat{B}_{\mathcal{I}}
$$

In terms of this, we define the linear impulsive system (1) restricted to $\mathcal{R}$ as

$$
\begin{aligned}
\dot{\xi}(t) & =\hat{A}_{\mathcal{C}} \xi(t)+\hat{B}_{\mathcal{C}} u(t) & t & \in \mathbb{R} \backslash \mathcal{T} \\
\xi\left(\tau_{k}\right) & =\hat{A}_{\mathcal{I}} \xi\left(\tau_{k}^{-}\right)+\hat{B}_{\mathcal{I}} w[k] & \tau_{k} & \in \mathcal{T}
\end{aligned}
$$

It is not difficult to show that for initial states related via $x\left(t_{0}\right)=R \xi\left(t_{0}\right)$, the state responses of (1) and (8) driven by the same input signals are related via $x(t)=R \xi(t), t \geq t_{0}$.

Next, we define the quotient space $\tilde{\mathcal{X}}=\mathcal{X} /\left\langle A_{\mathcal{C}}, A_{\mathcal{I}}\right\rfloor$ $\left.\mathcal{B}_{\mathcal{C}}+\mathcal{B}_{\mathcal{I}}\right\rangle$ together with the canonical projection $P: \mathcal{X} \rightarrow \tilde{\mathcal{X}}$ that satisfies $\operatorname{Ker} P=\left\langle A_{\mathcal{C}}, A_{\mathcal{I}} \mid \mathcal{B}_{\mathcal{C}}+\mathcal{B}_{\mathcal{I}}\right\rangle$. Again because $\left\langle A_{\mathcal{C}}, A_{\mathcal{I}} \mid \mathcal{B}_{\mathcal{C}}+\mathcal{B}_{\mathcal{I}}\right\rangle$ is invariant with respect to both $A_{\mathcal{C}}$ and $A_{\mathcal{I}}$, the following commutative relationships hold

$$
P A_{\mathcal{C}}=\tilde{A}_{\mathcal{C}} P \quad \text { and } P A_{\mathcal{I}}=\tilde{A}_{\mathcal{I}} P
$$

for well-defined $\tilde{A}_{\mathcal{C}}$ and $\tilde{A}_{\mathcal{I}}$ referred to as the maps induced in $\tilde{\mathcal{X}}$ by the associated map on $\mathcal{X}$. In addition, since Ker $P$ contains both $\mathcal{B}_{\mathcal{C}}$ and $\mathcal{B}_{\mathcal{I}}$,

$$
P B_{\mathcal{C}}=0 \quad \text { and } \quad P B_{\mathcal{I}}=0
$$

This leads to the unforced system induced in the quotient space $\tilde{\mathcal{X}}$ by the linear impulsive system (1), given as

$$
\begin{aligned}
\dot{\zeta}(t) & =\tilde{A}_{\mathcal{C}} \zeta(t) & t & \in \mathbb{R} \backslash \mathcal{T} \\
\zeta\left(\tau_{k}\right) & =\tilde{A}_{\mathcal{I}} \zeta\left(\tau_{k}^{-}\right) & \tau_{k} & \in \mathcal{T}
\end{aligned}
$$


with state transition matrix $\tilde{\Phi}(t, s)$. It can be shown that for initial states related via $\zeta\left(t_{0}\right)=P x\left(t_{0}\right)$ and any input signals, the state responses of (1) and (11) are related via $\zeta(t)=P x(t), t \geq t_{0}$.

The overall impulsive state equation (1) in the $(\xi, \zeta)$-coordinates is related to the subsystems (8) and (11) as follows. As $R$ is an injective map, the Moore-Penrose pseudoinverse $R^{\dagger}=\left(R^{T} R\right)^{-1} R^{T}$ gives a left inverse satisfying $R^{\dagger} R=I$. As $P$ is a surjective map, the Moore-Penrose pseudoinverse $P^{\dagger}=P^{T}\left(P P^{T}\right)^{-1}$ gives a right inverse satisfying $P P^{\dagger}=I$. By construction, $\operatorname{Ker} P=\operatorname{Im} R$, so that $P R=0$ and $R^{\dagger} P^{\dagger}=\left(R^{T} R\right)^{-1}(P R)^{T}\left(P P^{T}\right)^{-1}=0$.

From the orthogonal decomposition

$$
\mathcal{X}=\operatorname{Im} R \oplus[\operatorname{Im} R]^{\perp}=\operatorname{Im} R \oplus \operatorname{Im} P^{\dagger}
$$

any $x \in \mathcal{X}$ can be uniquely expressed $x=R \xi+P^{\dagger} \zeta$. This relationship can be inverted according to $\xi=R^{\dagger} x$ and $\zeta=$ $P x$.

The relationships (6), (7), (9), (10) along with the definitions $\bar{A}_{\mathcal{C}}=R^{\dagger} A_{\mathcal{C}} P^{\dagger}, \bar{A}_{\mathcal{I}}=R^{\dagger} A_{\mathcal{I}} P^{\dagger}$ combine to yield

$$
\begin{gathered}
{\left[\begin{array}{c}
\dot{\xi}(t) \\
\dot{\zeta}(t)
\end{array}\right]=\left[\begin{array}{cc}
\hat{A}_{\mathcal{C}} & \bar{A}_{\mathcal{C}} \\
0 & \tilde{A}_{\mathcal{C}}
\end{array}\right]\left[\begin{array}{l}
\xi(t) \\
\zeta(t)
\end{array}\right]+\left[\begin{array}{c}
\hat{B}_{\mathcal{C}} \\
0
\end{array}\right] u(t)} \\
t \in \mathbb{R} \backslash \mathcal{T} \\
{\left[\begin{array}{c}
\dot{\xi}\left(\tau_{k}\right) \\
\dot{\zeta}\left(\tau_{k}\right)
\end{array}\right]=\left[\begin{array}{cc}
\hat{A}_{\mathcal{I}} & \bar{A}_{\mathcal{I}} \\
0 & \tilde{A}_{\mathcal{I}}
\end{array}\right]\left[\begin{array}{c}
\xi\left(\tau_{k}^{-}\right) \\
\zeta\left(\tau_{k}^{-}\right)
\end{array}\right]+\left[\begin{array}{c}
\hat{B}_{\mathcal{I}} \\
0
\end{array}\right] w[k]} \\
\tau_{k} \in \mathcal{T}
\end{gathered}
$$

Following tradition for LTI systems, we loosely refer to the $\xi$-subsystem as the reachable subsystem and the $\zeta$-subsystem, being completely disconnected from the input signals, as the unreachable subsystem.

\section{B. Strong Reachability and Feedback Stabilization}

In general, the reachable set (3) and the invariant subspace $\left\langle A_{\mathcal{C}}, A_{\mathcal{I}} \mid \mathcal{B}_{\mathcal{C}}+\mathcal{B}_{\mathcal{I}}\right\rangle$ satisfy

$$
\mathcal{R}_{\text {fixed }}\left(t_{0}, t_{f}, \mathcal{T}\right) \subset\left\langle A_{\mathcal{C}}, A_{\mathcal{I}} \mid \mathcal{B}_{\mathcal{C}}+\mathcal{B}_{\mathcal{I}}\right\rangle \subset \mathcal{X}
$$

with possibly strict subspace containments. The following definition characterizes the situation in which the reachable set is the entire state space uniformly with respect to the impulse time set and any time interval containing a requisite number of impulse times.

Definition 3.1: (Strong Reachability) The impulsive system (1) is strongly reachable if there exists a positive integer $\ell$ such that for all $\mathcal{T}$ satisfying Assumption 2.1, $\mathcal{R}_{\text {fixed }}\left(t_{0}, t_{f}, \mathcal{T}\right)=\mathcal{X}$ for any finite interval $\left(t_{0}, t_{f}\right)$ containing at least $\ell$ impulse times in $\mathcal{T}$.

As a consequence of [8, Lemma 2.7], strong reachability is equivalent to boundedness and positive definiteness of $W\left(t, \sigma_{\ell+2}(t)\right)$ uniformly in $t$ and $\mathcal{T}$ satisfying Assumption 2.1 and is further shown in [8] to be sufficient to achieve exponential stabilization via state feedback. The development therein does not require the impulsive system to be reversible, i.e., $A_{\mathcal{I}}$ invertible, at the expense of added complexity in the analysis. On the other hand, the systems under consideration in [8] did not include the discrete-time input signal.
It was subsequently established in [9] that the following geometric conditions

$$
\left\langle A_{\mathcal{C}}, A_{\mathcal{I}} \mid \mathcal{B}_{\mathcal{C}}+\mathcal{B}_{\mathcal{I}}\right\rangle=\mathcal{X}, \quad \mathcal{A}_{\mathcal{I}}+\mathcal{B}_{\mathcal{I}}+\left\langle A_{\mathcal{C}} \mid \mathcal{B}_{\mathcal{C}}\right\rangle=\mathcal{X}
$$

(where $\mathcal{A}_{\mathcal{I}}:=\operatorname{Im} A_{\mathcal{I}}$ ) are necessary and almost sufficient for strong reachability. The possible existence of pathological impulse time sets for which $\mathcal{R}_{\text {fixed }}\left(t_{0}, t_{f}, \mathcal{T}\right) \neq \mathcal{X}$ for some time intervals containing the requisite number of impulse times thwarts the true sufficiency of (13). It is also shown in [9] that the second condition in (13) is equivalent to feedback reversibility of (1), that is, the ability to achieve reversibility via the application of a suitable state feedback law. As such, strong reachability implies feedback reversibility. Moreover, the application of state feedback that achieves reversibility does not alter the reachable set, and so it is possible without any real loss of generality to consider exponential stabilization via state feedback for strongly reachable systems in the reversible case.

Here we provide alternate formulae for stabilizing feedback gains that incorporate the discrete-time input signal and for which the resulting closed-loop system is susceptible to a more direct stability analysis than that conducted in [8]. A straightforward adaptation of [8, Lemmas 2.7 and 2.9] indicates that a strongly reachable reversible impulsive state equation (1) (with associated integer $\ell$ ) has a weighted controllability gramian

$$
\begin{gathered}
W_{\alpha}\left(t_{0}, t_{f}\right)=\int_{t_{0}}^{t_{f}} e^{2 \alpha\left(t_{0}-\tau\right)} \Phi\left(t_{0}, \tau\right) B_{\mathcal{C}} B_{\mathcal{C}}^{T} \Phi^{T}\left(t_{0}, \tau\right) d \tau \\
+\sum_{j=\kappa\left(t_{0}\right)+1}^{\kappa\left(t_{f}\right)} e^{2 \alpha\left(t_{0}-\tau_{j}\right)} \Phi\left(t_{0}, \tau_{j}\right) B_{\mathcal{I}} B_{\mathcal{I}}^{T} \Phi^{T}\left(t_{0}, \tau_{j}\right)
\end{gathered}
$$

with $\alpha$ a finite, positive parameter, for which $W_{\alpha}\left(t, \sigma_{\ell+2}(t)\right)$ is bounded and positive definite uniformly in $t$ and $\mathcal{T}$ satisfying Assumption 2.1, implying the same for $W_{\alpha}^{-1}\left(t, \sigma_{\ell+2}(t)\right)$.

In terms of this, the state feedback law

$$
u(t)=K_{\mathcal{C}}(t) x(t) \quad w[k]=K_{\mathcal{I}}[k] x\left(\tau_{k}^{-}\right)
$$

for bounded feedback gains

$$
\begin{aligned}
& K_{\mathcal{C}}(t)=-B_{\mathcal{C}}^{T} W_{\alpha}^{-1}\left(t, \sigma_{\ell+2}(t)\right) \\
& K_{\mathcal{I}}[k]=-B_{\mathcal{I}}^{T} A_{\mathcal{I}}^{-1} W_{\alpha}^{-1}\left(\tau_{k}^{-}, \tau_{k+\ell+2}^{-}\right)
\end{aligned}
$$

with $K_{\mathcal{C}}(t)$ piecewise continuous, yields an exponentially stable closed-loop state equation

$$
\begin{aligned}
\dot{x}(t) & =\left(A_{\mathcal{C}}+B_{\mathcal{C}} K_{\mathcal{C}}(t)\right) x(t) & t & \in \mathbb{R} \backslash \mathcal{T} \\
x\left(\tau_{k}\right) & =\left(A_{\mathcal{I}}+B_{\mathcal{I}} K_{\mathcal{I}}[k]\right) x\left(\tau_{k}^{-}\right) & \tau_{k} & \in \mathcal{T}
\end{aligned}
$$

The feedback gain construction (16) is motivated by results for time-varying linear systems in both continuous-time and discrete-time cases [2].

\section{StabilizABILITY}

In this section we present three stabilizability properties that turn out to be equivalent under the following assumption:

Assumption 4.1: The linear impulsive system (1) restricted to $\mathcal{R}$ given by (8) is strongly reachable. 
A useful consequence of Assumption 4.1 is the existence of a nonnegative integer $\ell$ and a finite, positive real $\hat{\lambda}_{\text {min }}$ for which $(R \xi)^{T} W\left(\sigma_{-\ell-2}(t), t\right)(R \xi) \geq \hat{\lambda}_{\min }\|R \xi\|^{2}$ for all $\xi \in \mathcal{R}$ and all $t$ and $\mathcal{T}$ satisfying Assumption 2.1.

The first stabilizability property is inspired by the definition of uniform stabilizability introduced in [1] for timevarying discrete-time linear systems and later invoked in [3].

Property 4.2: For the impulsive system (1) there exist finite, nonnegative integers $p, m$, nonnegative real $d<1$, and finite, positive real $b$ such that whenever

$$
\left\|\Phi^{T}\left(t, \sigma_{-m}(t)\right) x\right\| \geq d\|x\|
$$

for some $x \in \mathcal{X}$ and $t \in \mathbb{R}$, then

$$
x^{T} W\left(\sigma_{-p}(t), t\right) x \geq b\|x\|^{2}
$$

The second property is frequently adopted as the defining characterization of stabilizability, namely the existence of an exponentially stabilizing state feedback law.

Property 4.3: For the impulsive system (1) there exists a state feedback law (15) with bounded feedback gain matrices $K_{\mathcal{C}}(t)$ and $K_{\mathcal{I}}[k]$, with the former piecewise continuous, for which the closed-loop state equation (17) is exponentially stable.

And finally, the following property generalizes the familiar notion for LTI systems that the 'uncontrollable' subsystem is exponentially stable.

Property 4.4: The unforced system (11) induced in the quotient space $\tilde{\mathcal{X}}$ by the linear impulsive system (1) is exponentially stable.

The main results of the paper now establish, under Assumption 4.1, the equivalence of these three properties. First the equivalence of Properties 4.2 and 4.4 is shown, followed by the equivalence of Properties 4.3 and 4.4 .

Theorem 4.5: For the linear impulsive state equation (1) satisfying Assumption 2.1, Property 4.2 implies Property 4.4. In addition, under Assumption 4.1, Property 4.4 implies Property 4.2 .

Proof. We first show that Property 4.2 implies Property 4.4. First,

$$
\begin{aligned}
\operatorname{Im} P^{T}=\langle & \left\langle A_{\mathcal{C}}, A_{\mathcal{I}} \mid \mathcal{B}_{\mathcal{C}}+\mathcal{B}_{\mathcal{I}}\right\rangle^{\perp} \\
& \subset \mathcal{R}_{\text {fixed }}^{\perp}\left(\sigma_{-p}(t), t, \mathcal{T}\right)=\operatorname{Ker} W\left(\sigma_{-p}(t), t\right)
\end{aligned}
$$

and so for any $0 \neq \zeta \in \tilde{\mathcal{X}}$ and any $t \in \mathbb{R}$, $\left(P^{T} \zeta\right)^{T} W\left(\sigma_{p}(t), t\right)\left(P^{T} \zeta\right)=0$. As a consequence, for any $0 \neq \zeta \in \tilde{\mathcal{X}}$ and any $t \in \mathbb{R}$ there must hold

$$
\left\|\Phi^{T}\left(t, \sigma_{-m}(t)\right) P^{T} \zeta\right\|<d\left\|P^{T} \zeta\right\|
$$

We next claim that for any $0 \neq \zeta \in \tilde{\mathcal{X}}, t \in \mathbb{R}$, and positive integer $r$, there must hold $\left\|\Phi^{T}\left(t, \sigma_{-r m}(t)\right) P^{T} \zeta\right\|<$ $d^{r}\left\|P^{T} \zeta\right\|$ The inequality clearly holds for $r=1$. We now assume that the inequality holds for any $0 \neq \zeta \in \tilde{\mathcal{X}}$, any $t \in$ $\mathbb{R}$, and some $r>1$. From the commutative relationships (9) it follows that $P \Phi(\cdot, \cdot)=\tilde{\Phi}(\cdot, \cdot) P$. Upon taking transposes through this identity

$$
\begin{aligned}
\Phi^{T}\left(t, \sigma_{-r m}(t)\right) P^{T} \zeta=P^{T} \tilde{\Phi}^{T}\left(t, \sigma_{-r m}(t)\right) \zeta & \\
& \in \operatorname{Ker} W\left(\sigma_{-p}(t), t\right)
\end{aligned}
$$

and so (18) applies to $\Phi^{T}\left(t, \sigma_{-r m}(t)\right) P^{T} \zeta$ and $\sigma_{-r m}(t) \in \mathbb{R}$ yielding

$$
\begin{aligned}
&\left\|\Phi^{T}\left(t, \sigma_{-(r+1) m}(t)\right) P^{T} \zeta\right\|= \| \Phi^{T}\left(\sigma_{-r m}(t), \sigma_{-(r+1) m}(t)\right) \\
& \quad \times \Phi^{T}\left(t, \sigma_{-r m}(t)\right) P^{T} \zeta \| \\
&<d\left\|\Phi^{T}\left(t, \sigma_{-r m}(t)\right) P^{T} \zeta\right\| \\
&<d d^{r}\left\|P^{T} \zeta\right\|=d^{r+1}\left\|P^{T} \zeta\right\|
\end{aligned}
$$

thereby establishing the claim.

Thus, for any $0 \neq \zeta \in \tilde{\mathcal{X}}$ and any $t \in \mathbb{R}$

$$
\left\|P^{T} \tilde{\Phi}^{T}\left(t, \sigma_{-r m}(t)\right) \zeta\right\|<d^{r}\left\|P^{T} \zeta\right\|
$$

Using $\left(P P^{\dagger}\right)^{T}=I$, standard inequalities involving the spectral matrix norm give

$$
\left\|\tilde{\Phi}\left(t, \sigma_{-r m}(t)\right)\right\|=\left\|\tilde{\Phi}^{T}\left(t, \sigma_{-r m}(t)\right)\right\| \leq\|P\|\left\|P^{\dagger}\right\| d^{r}
$$

for any $t \in \mathbb{R}$.

We proceed to show that the unforced system (11) is exponentially stable. First, because

$$
r m \underline{\delta} \leq t-\sigma_{-r m}(t) \leq r m \bar{\delta}
$$

choosing $\tilde{\lambda}$ so that $e^{-\tilde{\lambda} m \bar{\delta}}=d$ yields

$$
\begin{aligned}
\left\|\tilde{\Phi}\left(t, \sigma_{-r m}(t)\right)\right\| \leq\|P\|\left\|P^{\dagger}\right\| e^{-r m \bar{\delta} \hat{\lambda}} & \leq\|P\|\left\|P^{\dagger}\right\| e^{-\hat{\lambda}\left(t-\sigma_{-r m}(t)\right)}
\end{aligned}
$$

for any $r \geq 1$ and $t \geq t_{0}$.

For any $t$ and $s<t$, let $r$ denote the least nonnegative integer for which $\sigma_{-(r+1) m}(t) \leq s<\sigma_{-r m}(t)$ and $\tilde{\Phi}(t, s)=\tilde{\Phi}\left(t, \sigma_{-r m}(t)\right) \tilde{\Phi}\left(\sigma_{-r m}(t), s\right)$. The right factor is norm bounded by the finite constant $\tilde{M}_{m}:=$ $\exp \left[\left\|\tilde{A}_{\mathcal{C}}\right\| m \bar{\delta}\right]\left\|\tilde{A}_{\mathcal{I}}\right\|_{\tilde{x}^{m}}^{m}$ uniformly in $s$ and $t$. Thus, by taking $\tilde{k}=\tilde{M}_{m}\|P\|\left\|P^{\dagger}\right\| e^{\tilde{\lambda} m \bar{\delta}}$ we obtain

$$
\begin{aligned}
\|\tilde{\Phi}(t, s)\| & \leq\left\|\tilde{\Phi}\left(t, \sigma_{-r m}(t)\right)\right\|\left\|\Phi\left(\sigma_{-r m}(t), s\right)\right\| \\
& \leq \tilde{M}_{m}\|P\|\left\|P^{\dagger}\right\| e^{-\tilde{\lambda}\left(t-\sigma_{-r m}(t)\right)} \\
& =\tilde{M}_{m}\|P\|\left\|P^{\dagger}\right\| e^{\tilde{\lambda}\left(\sigma_{-r m}(t)-s\right)} e^{-\tilde{\lambda}(t-s)} \\
& \leq \tilde{M}_{m}\|P\|\left\|P^{\dagger}\right\| e^{\tilde{\lambda} m \bar{\delta}} e^{-\tilde{\lambda}(t-s)} \\
& =\tilde{k} e^{-\tilde{\lambda}(t-s)}
\end{aligned}
$$

from which it follows that (11) is exponentially stable.

We next show that, under Assumption 4.1, Property 4.4 implies Property 4.2. Suppose that Property 4.2 does not hold. That is, corresponding to any finite, nonnegative integers $p, m$, nonnegative real $d<1$, and finite, positive real $b$ there exist $0 \neq x \in \mathcal{X}$ and $t \in \mathbb{R}$ for which

$$
\left\|\Phi^{T}\left(t, \sigma_{-m}(t)\right) x\right\| \geq d\|x\|
$$

and

$$
x^{T} W\left(\sigma_{-p}(t), t\right) x<b\|x\|^{2}
$$

We further assume that the unforced system (11) is exponentially stable and proceed to establish a contradiction. Under this assumption, there exist finite constants $\tilde{k} \geq 1$ and $\tilde{\lambda}>0$ for which

$$
\left\|\tilde{\Phi}\left(t, \sigma_{-m}(t)\right)\right\|=\left\|\tilde{\Phi}^{T}\left(t, \sigma_{-m}(t)\right)\right\| \leq \tilde{k} e^{-\tilde{\lambda}\left(t-\sigma_{-m}(t)\right)}
$$


for all $t \in \mathbb{R}$, and nonnegative integers $m$. Given $\epsilon \in(0,1)$, we choose $m$ large enough to ensure that $\|P\|\left\|{ }_{\tilde{\lambda}}\right\| P^{\dagger} \| \tilde{k} e^{-\tilde{\lambda} m \underline{\delta}}$ $<1-\epsilon$ and select $d \in\left(\epsilon+\|P\|\left\|P^{\dagger}\right\| \tilde{k} e^{-\tilde{\lambda} m \underline{\delta}}, 1\right)$.

Any $x \in \mathcal{X}$ can also be uniquely decomposed as $x=$ $R \xi+P^{T} \zeta$ and, under Assumption 4.1 with $p=\ell+2$,

$x^{T} W\left(\sigma_{-p}(t), t\right) x=(R \xi)^{T} W\left(\sigma_{-p}(t), t\right)(R \xi) \geq \hat{\lambda}_{\min }\|R \xi\|^{2}$

As a consequence of Assumption 2.1, we have the bound $\left\|\Phi^{T}\left(t, \sigma_{-m}(t)\right)\right\|=\left\|\Phi\left(t, \sigma_{-m}(t)\right)\right\| \leq M_{m} \quad$ for all $\quad t \in \mathbb{R}$ for a finite, positive number $M_{m}$ depending on $m$. With $p$, $m$, and $d$ fixed as characterized above along with positive $b \leq \hat{\lambda}_{\min }\left(\epsilon / M_{m}\right)^{2}$, let $0 \neq x \in \mathcal{X}$ and $t \in \mathbb{R}$ satisfy (19) and (20). Then $\|R \xi\|<\left(\epsilon / M_{m}\right)\|x\|$ and

$$
\begin{aligned}
& \left\|\Phi^{T}\left(t, \sigma_{-m}(t)\right) x\right\| \\
& \quad \leq\left\|\Phi^{T}\left(t, \sigma_{-m}(t)\right) R \xi\right\|+\left\|\Phi^{T}\left(t, \sigma_{-m}(t)\right) P^{T} \zeta\right\| \\
& \quad=\left\|\Phi^{T}\left(t, \sigma_{-m}(t)\right) R \xi\right\|+\left\|P^{T} \tilde{\Phi}^{T}\left(t, \sigma_{-m}(t)\right) \zeta\right\| \\
& \quad \leq M_{m}\|R \xi\|+\|P\| \tilde{k} e^{-\tilde{\lambda}\left(t-\sigma_{-m}(t)\right)}\|\zeta\| \\
& \quad<\epsilon\|x\|+\|P\| \tilde{k} e^{-\tilde{\lambda} m \underline{\delta}}\|\zeta\| \\
& \quad \leq \epsilon\|x\|+\|P\|\left\|P^{\dagger}\right\| \tilde{k} e^{-\tilde{\lambda} m \underline{\delta}}\left\|P^{T} \zeta\right\| \\
& \quad \leq\left(\epsilon+\|P\|\left\|P^{\dagger}\right\| \tilde{k} e^{-\tilde{\lambda} m \underline{\delta}}\right)\|x\|
\end{aligned}
$$

which, because we have chosen $d>\epsilon+\|P\|\left\|P^{\dagger}\right\| \tilde{k} e^{-\tilde{\lambda} m \underline{\delta}}$, contradicts (19) and completes the proof.

Theorem 4.6: For the linear impulsive state equation (1) satisfying Assumption 2.1, Property 4.3 implies Property 4.4. In addition, under Assumption 4.1, Property 4.4 implies Property 4.3.

Proof. We first show that Property 4.3 implies Property 4.4. Suppose there exists a state feedback law (15) for which the closed-loop impulsive state equation (17) is exponentially stable. With $\Phi_{\mathrm{CL}}(\cdot, \cdot)$ denoting the state transition matrix for (17), there exist finite $k \geq 1$ and $\lambda>0$ satisfying $\left\|\Phi_{\mathrm{CL}}\left(t, t_{0}\right)\right\| \leq k e^{-\lambda\left(t-t_{0}\right)}$ for all $t_{0}$ and $t \geq t_{0}$. From the relationships (9), (10) we have for any feedback gains $K_{\mathcal{C}}(t)$, $K_{\mathcal{I}}[k]$

$$
P\left(A_{\mathcal{C}}+B_{\mathcal{C}} K_{\mathcal{C}}(t)\right)=\tilde{A}_{\mathcal{C}} P, \quad P\left(A_{\mathcal{I}}+B_{\mathcal{I}} K_{\mathcal{I}}[k]\right)=\tilde{A}_{\mathcal{I}} P
$$

which yields $P \Phi_{\mathrm{CL}}(\cdot, \cdot)=\tilde{\Phi}(\cdot, \cdot) P$ and so $\tilde{\Phi}(\cdot, \cdot)=$ $P \Phi_{\mathrm{CL}}(\cdot, \cdot) P^{\dagger}$. It follows immediately that

$$
\left\|\tilde{\Phi}\left(t, t_{0}\right)\right\| \leq k\|P\|\left\|P^{\dagger}\right\| e^{-\lambda\left(t-t_{0}\right)}
$$

for all $t_{0}$ and $t \geq t_{0}$ implying that the unforced system (11) is exponentially stable.

We next show that under Assumption 4.1, Property 4.4 implies Property 4.3. The stabilizing state feedback law construction discussed Section III-B applied to the strongly reachable restricted system (8) yields the existence of a state feedback law

$$
u(t)=\hat{K}_{\mathcal{C}}(t) \xi(t) \quad w[k]=\hat{K}_{\mathcal{I}}[k] \xi\left(\tau_{k}^{-}\right)
$$

for which

$$
\begin{aligned}
\dot{\xi}(t) & =\left(\hat{A}_{\mathcal{C}}+\hat{B}_{\mathcal{C}} \hat{K}_{\mathcal{C}}(t)\right) \xi(t) & t & \in \mathbb{R} \backslash \mathcal{T} \\
\xi\left(\tau_{k}\right) & =\left(\hat{A}_{\mathcal{I}}+\hat{B}_{\mathcal{I}} \hat{K}_{\mathcal{I}}[k]\right) \xi\left(\tau_{k}^{-}\right) & \tau_{k} & \in \mathcal{T}
\end{aligned}
$$

is exponentially stable. The overall closed-loop state equation in the $(\xi, \zeta)$-coordinates

$$
\begin{aligned}
{\left[\begin{array}{c}
\dot{\xi}(t) \\
\dot{\zeta}(t)
\end{array}\right] } & =\left[\begin{array}{cc}
\hat{A}_{\mathcal{C}}+\hat{B}_{\mathcal{C}} \hat{K}_{\mathcal{C}}(t) & \bar{A}_{\mathcal{C}} \\
0 & \tilde{A}_{\mathcal{C}}
\end{array}\right]\left[\begin{array}{l}
\xi(t) \\
\zeta(t)
\end{array}\right] \quad t \in \mathbb{R} \backslash \mathcal{T} \\
{\left[\begin{array}{c}
\dot{\xi}\left(\tau_{k}\right) \\
\dot{\zeta}\left(\tau_{k}\right)
\end{array}\right] } & =\left[\begin{array}{cc}
\hat{A}_{\mathcal{I}}+\hat{B}_{\mathcal{I}} \hat{K}_{\mathcal{I}}[k] & \bar{A}_{\mathcal{I}} \\
0 & \tilde{A}_{\mathcal{I}}
\end{array}\right]\left[\begin{array}{l}
\xi\left(\tau_{k}^{-}\right) \\
\zeta\left(\tau_{k}^{-}\right)
\end{array}\right] \quad \tau_{k} \in \mathcal{T}
\end{aligned}
$$

consists of, by Property 4.4, the cascade interconnection of two exponentially stable subsystems with bounded coupling and is therefore exponentially stable. From this, the gains $K_{\mathcal{C}}(t)=\hat{K}_{\mathcal{C}}(t) R^{\dagger}$ and $K_{\mathcal{I}}[k]=\hat{K}_{\mathcal{I}}[k] R^{\dagger}$ yield an exponentially stabilizing state feedback law in the $x$-coordinates (15), so that Property 4.3 holds.

Thus, exponential stability of the 'unreachable' subsystem (11) is necessary and sufficient to achieve closed-loop exponential stability via state feedback and so, as with LTI systems, full state reachability of a linear impulsive system is not necessary for state feedback stabilization.

\section{Application: Synchronization of Chaotic SYSTEMS}

Here we apply the ideas of the preceding section to the much-studied problem of synchronizing two Lorenz oscillators using impulsive control [5], [6]. A Lorenz oscillator is modelled by the state equation

$$
\begin{aligned}
& \dot{x}(t)=-\sigma x(t)+\sigma y(t) \\
& \dot{y}(t)=\rho x(t)-y(t)-x(t) z(t) \\
& \dot{z}(t)=x(t) y(t)-\beta z(t)
\end{aligned}
$$

which exhibits a strange attractor for the parameter values $\sigma=10, \rho=28$, and $\beta=8 / 3$.

The synchronization problem involves regulating the error between two Lorenz oscillators, one with state $X(t)=$ $(x(t), y(t), z(t))$ (the drive system) and another with state $\tilde{X}(t)=(\tilde{x}(t), \tilde{y}(t), \tilde{z}(t))$ (the driven system). Synchronization is to be achieved at the driven system using measurements of the drive system state at a set of discrete-time instants $\mathcal{T}=\left\{\tau_{k}, k \geq 1\right\}$. These time instants are assumed to satisfy $\tau_{1}-t_{0}=\delta_{\text {even }}$ and

$$
\tau_{2 j}-\tau_{2 j-1}=\delta_{\text {odd }} \quad \tau_{2 j+1}-\tau_{2 j}=\delta_{\text {even }} \quad j \geq 1
$$

A control policy involving the error between the drive state and the driven state at these time instants is then used to effect an instantaneous jump in the driven state in order to regulate the synchronization error. Specifically, a control law of the form

$$
\tilde{X}\left(\tau_{k}\right)=\tilde{X}\left(\tau_{k}^{-}\right)-U\left[k, X\left(\tau_{k}^{-}\right)-\tilde{X}\left(\tau_{k}^{-}\right)\right]
$$

yields the impulsive error dynamics with state $e(t)=X(t)-$ $\tilde{X}(t)$ given by

$$
\begin{aligned}
\dot{e}(t) & =A e(t)+\Phi(X(t), e(t)) & t & \in \mathbb{R} \backslash \mathcal{T} \\
e\left(\tau_{k}\right) & =e\left(\tau_{k}^{-}\right)+U\left[k, e\left(\tau_{k}^{-}\right)\right] & \tau_{k} & \in \mathcal{T}
\end{aligned}
$$

in which

$$
A=\left[\begin{array}{rrr}
-\sigma & \sigma & 0 \\
\rho & -1 & 0 \\
0 & 0 & -\beta
\end{array}\right]
$$


and the nonlinear term is $\Phi(X, e)=\varphi(X)-\varphi(X+e)$ with $\varphi(X)=\left[\begin{array}{lll}0 & -x z & x y\end{array}\right]^{T}$. In what follows, we simply treat the nonlinear term $\Phi(X(t), e)$ as a vanishing perturbation that is locally Lipschitz in $e$ and focus on the underactuated linear error dynamics

$$
\begin{aligned}
\dot{e}(t) & =A e(t) & t & \in \mathbb{R} \backslash \mathcal{T} \\
e\left(\tau_{k}\right) & =e\left(\tau_{k}^{-}\right)+B w[k] & \tau_{k} & \in \mathcal{T}
\end{aligned}
$$

together with state feedback law $w[k]=K_{\mathcal{I}}[k] e\left(\tau_{k}^{-}\right)$that only influences the first component of the error state so that $B=\left[\begin{array}{lll}1 & 0 & 0\end{array}\right]^{T}$. Thus, (24) has the form of the linear impulsive state equation (1) with data $A_{\mathcal{C}}=A, B_{\mathcal{C}}=0_{3 \times 1}$, $A_{\mathcal{I}}=I_{3 \times 3}$, and $B_{\mathcal{I}}=B$.

Proceeding with the stabilizability analysis, it is not hard to show that

$$
\left\langle A_{\mathcal{C}}, A_{\mathcal{I}} \mid \mathcal{B}_{\mathcal{C}}+\mathcal{B}_{\mathcal{I}}\right\rangle=\operatorname{Im}\left[\begin{array}{ll}
B & A B
\end{array}\right]=\operatorname{Im}\left[\begin{array}{rr}
1 & -\sigma \\
0 & \rho \\
0 & 0
\end{array}\right]
$$

We observe that (24) is already in the form of the decomposition (12) with the identifications $\xi \leftrightarrow\left(e_{x}, e_{y}\right)$ and $\zeta \leftrightarrow e_{z}$. Moreover, it is straightforward to verify that the system restricted to $\mathcal{R}$ given by (8) characterized by

$\hat{A}_{\mathcal{C}}=\left[\begin{array}{rr}-\sigma & \sigma \\ \rho & -1\end{array}\right], \hat{B}_{\mathcal{C}}=0_{2 \times 1}, \hat{A}_{\mathcal{I}}=I_{2 \times 2}, \hat{B}_{\mathcal{I}}=\left[\begin{array}{l}1 \\ 0\end{array}\right]$

is strongly reachable with $\ell=1$ for any impulse time set. Finally, we note that with $\tilde{A}_{\mathcal{C}}=-\beta<0$ and $\tilde{A}_{\mathcal{I}}=1$, the subsystem (11) is easily seen to be exponentially stable.

The construction of a stabilizing gain $K_{\mathcal{I}}[k]$ proceeds as follows. First, because $\hat{A}_{\mathcal{I}}=I_{2 \times 2}$, the subsystem (8) is trivially reversible and, further, $\hat{\Phi}(t, s)=\exp \left[\hat{A}_{\mathcal{C}}(t-s)\right]$. Thus, using $\ell=1$

$$
\begin{aligned}
& \hat{W}_{\alpha}\left(\tau_{k}^{-}, \tau_{k+\ell+2}^{-}\right)= \\
& \sum_{j=k}^{k+2} e^{\left(\alpha I+\hat{A}_{\mathcal{C}}\right)\left(\tau_{k}-\tau_{j}\right)} \hat{B}_{\mathcal{I}} \hat{B}_{\mathcal{I}}^{T} e^{\left(\alpha I+\hat{A}_{\mathcal{C}}\right)^{T}\left(\tau_{k}-\tau_{j}\right)}
\end{aligned}
$$

for finite, positive $\alpha$ which leads to

$$
K_{\mathcal{I}}[k]=-\left[\left[\begin{array}{ll}
1 & 0
\end{array}\right] \hat{W}_{\alpha}^{-1}\left(\tau_{k}^{-}, \tau_{k+\ell+2}^{-}\right) \mid 0\right]
$$

We observe that the $z$-component of the drive state need not be transmitted to the driven system at the impulse times due to the zero in the third component of this feedback gain.

For explicit numerical computations we adopt the impulse time spacing (22) with $\delta_{\text {even }}=0.05 \mathrm{~s}$ and $\delta_{\text {odd }}=0.1 \mathrm{~s}$. This along with $\alpha=0.5$ gives

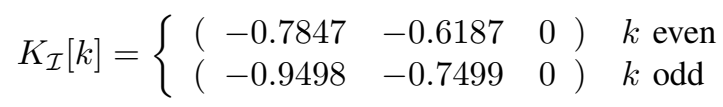

The associated state feedback law yields global exponential stability of the linear error dynamics and, via standard perturbation arguments, local exponential stability of the nonlinear error dynamics. Nonlinear error responses for $X(0)=\left[\begin{array}{lll}10 & -10 & 10\end{array}\right]^{T}$ and $\tilde{X}(0)=\left[\begin{array}{lll}0 & 0 & 0\end{array}\right]^{T}$ are shown in Fig. 1.

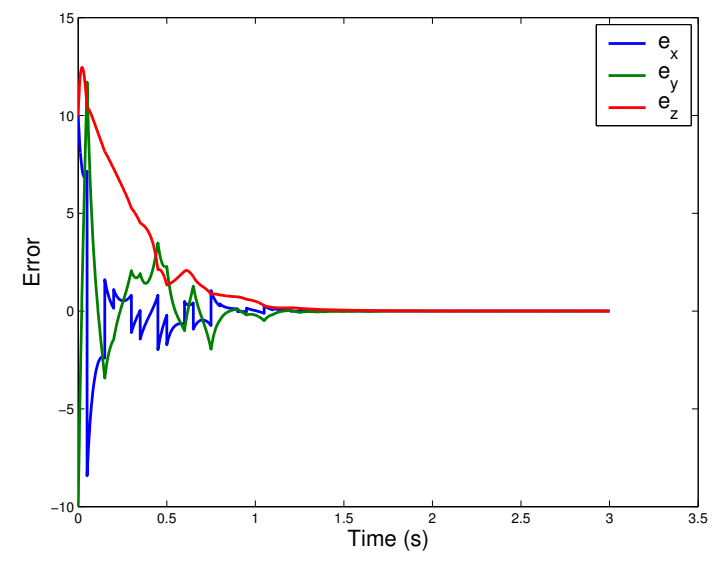

Fig. 1. Nonlinear error responses.

\section{CONCluding Remarks}

This paper has presented a stabilizability analysis for a class of linear impulsive systems. Three system properties resembling stabilizability conditions that have appeared in the literature for time-varying and time-invariant linear systems have been shown to be equivalent under mild technical assumptions. Most notably, exponential stability of an 'unreachable' subsystem is necessary and sufficient to achieve closed-loop exponential stability via state feedback, thus extending well-known facts for the LTI case to linear impulsive systems. Developing a comparable analysis for the dual property of detecability and exploring the combined role of stabilizability and detectability in output feedback stabilization and optimal control for linear impulsive systems is the focus of ongoing investigation.

\section{REFERENCES}

[1] B. D. O. Anderson and J. B. Moore. Detectability and stabilizability of time-varying discrete-time linear systems. SIAM Journal of Control and Optimization, 19(1):20-32, 1981.

[2] V. H. L. Cheng. A direct way to stabilize continuous-time and discretetime linear time-varying systems. IEEE Transactions on Automatic Control, AC-24(4):641-643, 1979.

[3] J.-W. Lee. Inequality-based properties of detectability and stabilizability of linear time-varying systems in discrete time. IEEE Transactions on Automatic Control, 54(3):634-641, 2009.

[4] J.-W. Lee and P. P. Khargonekar. Detectability and stabilizability of discrete-time switched linear systems. IEEE Transactions on Automatic Control, 54(3):424-437, 2009.

[5] Y Li. Some new less conservative criteria for impulsive synchronization of a hyperchaotic Lorenz system based on small impulsive signals. Nonlinear Analysis: Real World Applications, 11:713-719, 2010.

[6] Z. Li, Y. Soh, and C. Wen. Switched and Impulsive Linear Systems: Analysis, Design, and Applications. Springer-Verlag, 2005.

[7] E. A. Medina and D. A. Lawrence. Reachability and observability of linear impulsive systems. Automatica, 44:1304-1309, 2008.

[8] E. A. Medina and D. A. Lawrence. State feedback stabilization of linear impulsive systems. Automatica, 45:1476-1480, 2009.

[9] E. A. Medina and D. A. Lawrence. Feedback-reversibility and reachability of linear impulsive systems. Automatica, 46:1101-1106, 2010.

[10] W. M. Wonham. Linear Multivariable Control: A Geometric Approach. Springer-Verlag, New York, third edition edition, 1985.

[11] G. Xie and L. Wang. Controllability and stabilizability of switched linear-systems. Systems \& Control Letters, 48:135-155, 2003.

[12] G. Xie and L. Wang. Reachability realization and stabilizability of switched linear discrete-time systems. Journal of Mathematical Analysis and Applications, 280:209-220, 2003. 